\title{
Quantum weak-interaction-based measurement: from Sequential weak measurement to Protective Measurement
}

\author{
Enrico Rebufello ${ }^{\mathrm{a}, \mathrm{b}}$, Salvatore Virzi ${ }^{\mathrm{a}, \mathrm{c}}$, Fabrizio Piacentinia ${ }^{\mathrm{a}}$, Alessio Avella ${ }^{\mathrm{a}}$, Franco Zappa ${ }^{\mathrm{d}}$, \\ Rudi Lussana ${ }^{\mathrm{d}}$, Federica Villa ${ }^{\mathrm{d}}$, Alberto Tosi ${ }^{\mathrm{d}}$, Marco Gramegna ${ }^{\mathrm{a}}$, Giorgio Brida ${ }^{\mathrm{a}}$, Eliahu \\ Cohen $^{\mathrm{e}}$, Marco Barbieri ${ }^{\mathrm{f}}$, Ivo Pietro Degiovanni ${ }^{\mathrm{a}}$, and Marco Genovese ${ }^{\mathrm{a}}$ \\ aNRIM, Strada delle Cacce 91, I-10135, Torino, Italy \\ bPolitecnico di Torino, Corso Duca degli Abruzzi 24, I-10029, Torino, Italy \\ ${ }^{c}$ Dipartimento di Fisica, Università di Torino, via Giuria 1, I-10125, Torino, Italy \\ ${ }^{\text {d} P o l i t e c n i c o ~ d i ~ M i l a n o, ~ D i p a r t i m e n t o ~ d i ~ E l e t t r o n i c a, ~ I n f o r m a z i o n e ~ e ~ B i o i n g e g n e r i a, ~ P i a z z a ~}$ \\ Leonardo da Vinci 32, 20133, Milano, Italy \\ ePhysics Department, Centre for Research in Photonics, University of Ottawa, Advanced \\ Research Complex, 25 Templeton, Ottawa, ON, K1N 6N5, Canada \\ ${ }^{\mathrm{f}}$ Dipartimento di Scienze, Università degli Studi Roma Tre, Via della Vasca Navale 84, 00146 \\ Rome, Italy
}

\begin{abstract}
Weak value measurements have been a real breakthrough in the quantum measurement framework. In particular, quantum measurements may take advantage by anomalous weak values, i.e. values out of the eigenvalues spectrum of the measured observable, both for implementing new measurement techniques and studying Quantum Mechanics foundations. In this report we show three experiments with single photons presenting anomalous weak values: the first one tests the incompatibility between quantum mechanics and noncontextual hidden variables theories, the second one is the first realization of a sequential weak value evaluation of two incompatible observables on the same photon, and the last one shows how sequential weak values can be used to test Leggett-Garg inequalities extended to multiple-measurements scenarios.
\end{abstract}

Keywords: quantum optics, weak measurements, weak values

\section{INTRODUCTION}

In 1988 Aharonov, Albert and Vaidman introduced weak measurements, ${ }^{1}$ a new paradigm of quantum measurement where just a small amount of information is extracted from a single measurement, but the state does not collapse. In the same paper, they introduced the weak values, i.e., weak measurements of an operator performed on an ensemble of pre- and postselected states, which present some peculiar properties, e.g. assuming anomalous values (i.e., values outside the eigenvalue range of the observable). In recent years, weak values have been the subject of great interest thanks to the several applications ${ }^{2-13}$ found, such as the investigation of fundamental aspects of quantum mechanics as well as the possibility of amplifying the measurement of small parameter. ${ }^{14}$

In this paper we present three different experiments, useful both for the study of the foundations of quantum mechanics and for more practical applications.

\section{WEAK MEASUREMENTS AND WEAK VALUES}

In quantum mechanics, an indirect measurement describes a quantum measurement device (usually called meter $\mathcal{M}$ ), whose interaction with the system constitutes the measurement: by reading the meter one gets information about the value of the system observable. A special case of indirect measurements are weak measurements, ${ }^{15}$ consisting of two steps. In the first step the measurement device is coupled to the quantum system with a weak von Neumann coupling. ${ }^{16}$ In the second step the measurement device is strongly measured. The collapsed state of the measurement device is the outcome of the weak measurement process. 
For a measurement to be weak, the standard deviation of the measurement outcome should be larger than the difference between the eigenvalues of the system.

Let $|\phi\rangle$ denote the wavefunction of the measurement device. In the position basis, it can be written as:

$$
|\phi\rangle=\int_{x} \phi(x)|x\rangle d x
$$

where $x$ is the position variable of the measuring needle. The position operator of the needle is $\hat{X}: \hat{X}|x\rangle=x|x\rangle$. It is assumed that $\phi(x)$ behaves normally around 0 , with variance $\sigma^{2}: \phi(x)=e^{-x^{2} / 4 \sigma^{2}} /\left(2 \pi \sigma^{2}\right)$

Let $\mathcal{S}$ denote the system to be measured and suppose that $\hat{A}$ is an Hermitian operator of the system with eigenvectors $\left\{\left|a_{i}\right\rangle\right\}$ such that $\hat{A}\left|a_{i}\right\rangle=a_{i}\left|a_{i}\right\rangle$. Then, a general state vector for the system can be expressed as:

$$
|\psi\rangle=\sum_{i} \alpha_{i}\left|a_{i}\right\rangle
$$

The initial state of the total system, which comprises both the system and the meter, is $|\psi\rangle \otimes|\phi\rangle$ and the interaction Hamiltonian between the system and the meter is

$$
H_{\text {int }}=g \hat{A} \otimes \hat{P}
$$

with $g$ being the coupling constant and $\hat{P}$ being the observable canonically conjugated to $\hat{X}$.

It is easy to see that for each $\left|a_{i}\right\rangle, e^{-i g a_{i} \hat{P}}$ is the generator of the translations of $\hat{X}$. The system, then, evolves as:

$$
e^{-i H / \hbar}|\psi\rangle \otimes|\phi\rangle=\sum_{j} \alpha_{j}\left|a_{j}\right\rangle \otimes\left|\phi\left(x-g a_{j}\right)\right\rangle
$$

So, the initial state of the measuring needle, assumed centered in zero, is transformed in a superpositions of $\left|\phi_{j}\right\rangle=\left|\phi\left(x-g a_{j}\right)\right\rangle$ states. The strength of the interaction, ${ }^{17}$ then, can be quantified by the ratio $g / \sigma$. When $g / \sigma \gg 1$ the wavefunctions $\phi_{j}$ do not overlap and the measurement is strong. However, if $g / \sigma \ll 1$ the wavefunctions $\phi_{j}$ overlap each other. The lower the ratio, the weaker the measurement process.

After measuring the needle, the system's vector is slightly biased in a direction that corresponds to the needle's outcome value. On one hand, the information we get, that is the value of the needle, is very vague, on the other hand, the system's state does not collapse.

\subsection{WEAK VALUES}

Introducing a post-selection after the weak interaction results in the weak values, ${ }^{18}$ a new property of the system. Let $\hat{A}$ be an hermitian operator of the system $\mathcal{S}$ and let $|s\rangle$ and $|f\rangle$ be two states in the Hilbert space of the system. Let $|\phi\rangle$ be the initial state of the measuring device. Now suppose to perform a weak measurement of a particle in the initial state $|s\rangle$ using the interaction Hamiltonian (3) and then to post-select the final state $|f\rangle$. The overall probability amplitude is $\langle f|\hat{U}| s\rangle$ (with $\hat{U}=\exp \left(-i H_{\text {int }} / \hbar\right)$ ). This process can be described as a projective measurement of the system with the projector $\Pi=|f\rangle\langle f| \otimes \mathbb{1}_{\mathcal{M}}$. After the weak interaction and the post-selection, the state is: $|f\rangle\left\langle f\left|\exp \left(-i H_{\text {int }} / \hbar\right)\right| s\right\rangle \otimes|\phi\rangle$, that, for weak interaction $(g / \sigma \ll 1)$, can be approximated as:

$$
\begin{aligned}
& |f\rangle\left\langle f\left|\exp \left(-i H_{\text {int }} / \hbar\right)\right| s\right\rangle \otimes|\phi\rangle \approx|f\rangle\left\langle f\left|\left(1-\frac{i}{\hbar} g \hat{A} \otimes \hat{P}\right)\right| s\right\rangle \otimes|\phi\rangle= \\
& =|f\rangle\langle f \mid s\rangle\left(1-\frac{i}{\hbar}\langle A\rangle_{w} \hat{P}\right)|\phi\rangle \approx|f\rangle\langle f \mid s\rangle \exp \left(-\frac{i}{\hbar}\langle A\rangle_{w} \hat{P}\right)|\phi\rangle
\end{aligned}
$$

where

$$
\langle A\rangle_{w}=\frac{\langle f|\hat{A}| s\rangle}{\langle f \mid s\rangle}
$$

is the weak value. The meter wavefunction, consequently, changes into $\left|\phi^{\prime}\right\rangle=\left|\phi\left(x-g\langle A\rangle_{w}\right)\right\rangle$. 


\section{WEAK VALUES AND CONTEXTUALITY}

In recent years, a question emerged about anomalous weak values constituting a proof of the incompatibility of quantum theory with noncontextual hidden variables theories (NCHVTs). ${ }^{19}$ NCHVTs are theories that assume that a predetermined result of a particular measurement does notdepend on which other observables are simultaneously measured. Recently, it was demonstrated that, under specific experimental conditions, noncontextuality is incompatible with the observation of anomalous weak values. In the next section, this connection will be presented in the form of a theorem, and related experimental results will be shown.

\subsection{Theory}

The following theorem has been presented and demostrated in. ${ }^{20}$ Here, it will be presented in a form avoiding any reference to quantum mechanics.

Theorem - Suppose to have a preparation procedure $\mathcal{P}_{s}$, a strong measurement procedure $\mathcal{M}_{s}$ with outcomes OK or KO, and a nondestructive measurement procedure $\mathcal{M}_{W}$ with outcomes $x \in \mathbb{R}$, such that:

1. The pre- and post-selected states $|s\rangle$ and $|f\rangle$ are not orthogonal:

$$
p_{f}=P\left(O K \mid \mathcal{P}_{s}, \mathcal{M}_{s}\right)>0
$$

2. Ignoring the post-measurement state $\mathcal{M}_{W}$ is equivalent to a dichotomous measurement with unbiased noise:

$$
p\left(x \mid \mathcal{P}, \mathcal{M}_{W}\right)=p_{n}(x-g) P\left(1 \mid \mathcal{P}, \mathcal{M}_{A}\right)+p_{n}(x) P\left(0 \mid \mathcal{P}, \mathcal{M}_{A}\right) \forall \mathcal{P}
$$

for some strong measurement procedure $\mathcal{M}_{A}$ with outcomes 0 and 1 and probability distribution $F(x)$ with median $x=0$.

3. A "probability of disturbance" $p_{d}$ can be defined such that, ignoring the outcome of $\mathcal{M}_{W}$, it affects the post-selection in the same way as mixing it with another measurement:

$$
P\left(O K \mid \mathcal{P}, \mathcal{M}_{W}, \mathcal{M}_{f}=\left(1-p_{d}\right) P\left(O K \mid \mathcal{P}, \mathcal{M}_{f}\right)+p_{d} P\left(O K \mid \mathcal{P}, \mathcal{M}_{d}\right) \forall \mathcal{P}\right.
$$

for some measurement $\mathcal{M}_{d}$ with outcomes OK and KO.

4. The values of $x$ under pre- and post-selection have a negative bias that outweights $p_{d}$, that is, for $p_{-}=$ $\left(p_{|f\rangle}\right)^{-1} \int_{-\infty}^{0} P\left(x, O K \mid \mathcal{P}_{s}, \mathcal{M}_{W}, \mathcal{M}_{f}\right) d x$ holds:

$$
\mathcal{I}=p_{-}-\frac{1}{2}-\frac{p_{-}}{p_{f}}>0
$$

Then, there is no noncontextual ontological model for the preparation $\mathcal{P}_{s}$, the measurement $\mathcal{M}_{W}$ and the postselection $\mathcal{M}_{f}$ on OK satisfying the outcome determinism for strong measurements.

Here, we present the first experimental test of this theorem, performed by exploiting weak measurements on heralded single photons. ${ }^{21}$

In quantum mechanics, $\mathcal{P}$ corresponds to the preseletion of the polarization state $|s\rangle=\cos \theta|H\rangle+e^{i \beta} \sin \theta|V\rangle$ of a single photon, the post-selection is the projector $|f\rangle\langle f|$, which yelds to $p_{f}=P\left(O K \mid \mathcal{P}, \mathcal{M}_{f}\right)=|\langle f \mid s\rangle|^{2}$. The nondestructive measurement $\mathcal{M}_{W}$ is implemented as a weak interaction induced by the unitary evolution $\hat{U}=\exp (-i g \hat{A} \otimes \hat{P})$, where $g$ is the von Neumann coupling constant. 


\subsection{Experimental setup}

In the experiment ${ }^{22}$ (Fig. 1), a single photon state is prepared in the state $|s\rangle \otimes|\phi\rangle$, with $|\phi\rangle=\int_{x} d x F(x)|x\rangle$. Then, the single photon undergoes a weak interaction by interacting with a thin birifringent crystal, which induces a spatial walk-off. This interaction is described by the unitary transformation $\hat{U}$. The probability of finding the single photon in the position $x_{0}$ of the transverse plane is

$$
P\left(x_{0} \mid \mathcal{P}, \mathcal{M}_{W}\right)=\operatorname{tr}\left[M_{x_{0}}|\psi\rangle\langle\psi| M_{x_{0}}^{\dagger}\right]
$$

where $M_{x_{0}}|s\rangle=\left\langle x_{0}|\hat{U}| s\right\rangle|\phi\rangle . P\left(1 \mid \mathcal{P}, \mathcal{M}_{A}\right)$ and $P\left(0 \mid \mathcal{P}, \mathcal{M}_{A}\right)$ in Eq. (7) correspond, respectively, to the probability that the photon undergoes or does not the weak interaction. $\mathcal{M}_{d}$ ) in Eq. (8) represents an unknown measurement process. In order to demonstrare that its contribution is negligible because of the nondestructive nature of the measurement $\mathcal{M}_{W}$ (thanks to the weak interaction), one can exploit the parameter $p_{d}$, which is the disturbance that $\mathcal{M}_{W}$ causes to the subsequent strong measurement $\mathcal{M}_{f} \cdot p_{d}$ can be evaluated as the amount of decoherence induced on the single photon by the weak interaction $\hat{U}$ :

$$
p_{d}=1-e^{-\left(g^{2} / 4 \sigma^{2}\right)}
$$




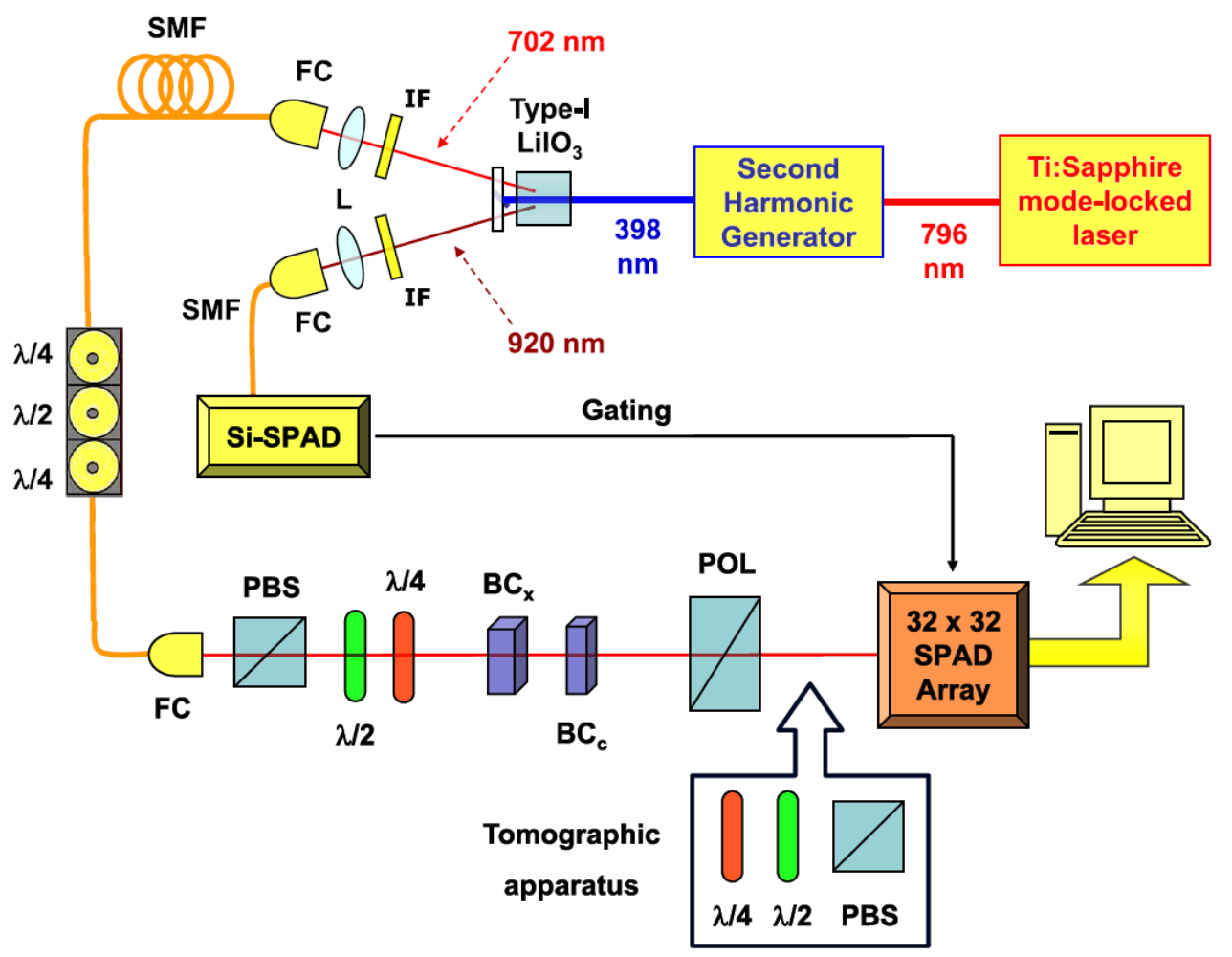

Figure 1: Experimental setup. The second harmonic emission of a $796 \mathrm{~nm}$ mode-locked Ti:sapphire laser (repetition rate: $76 \mathrm{MHz}$ pumps a $\mathrm{LiIO}_{3}$ nonlinear crystal in which type-I parametric down-conversion ${ }^{23,24}$ is produced. The idler photon $\left(\lambda_{i}=920 \mathrm{~nm}\right)$ is coupled to a single-mode fiber (SMF) and then detected by a single photon avalanche diode (SPAD), heralding the presence of the correlated signal photon $\left(\lambda_{s}=702 \mathrm{~nm}\right)$, which is SMF coupled and sent to the free-space optical path where the experiment is performed. The quality of the single-photon emission is given by an $\alpha$ value $^{25}$ of $0.13(1)$ without any background or dark-count subtraction. The initial state $|s\rangle$ of the signal photon is prepared by the interaction with a polarizing beam splitter (PBS), followed by an half-wave plate and a quarter-wave plate. Then, the photon interacts with a $1 \mathrm{~mm}$ long birifringet crystal $\left(\mathrm{BC}_{\mathrm{x}}\right)$, responsible for the weak interaction, whose extraordinary (e) optical axis lies in the $\mathrm{X}-\mathrm{Z}$ plane, with an angle of $\pi / 4$ with respect to the $\mathrm{Z}$ direction. This induces a spatial walk-off, which slightly separates the vertically polarized paths of the photons with respect to the horizontally polarized photons one, inducing a small decoherence (below $1 \%)$ on the initial state $|s\rangle$. The second crystal $\left(\mathrm{BC}_{\mathrm{c}}\right)$ is used to compensate the phase and time shift induced by the first one. After the weak interaction, the heralded single photon is projected onto the post-selected state $|f\rangle$ by a Glan linear polarizer and then adressed to a space-resolving single photon detector, a $32 x 32$ prototype SPAD Array. ${ }^{26}$ Furthermore, a removable polarization tomographic apparatus ${ }^{27,28}$ is inserted when needed to verify the fulfillment of the condition in Eq. (7).

\subsection{Results}

Fig. 2 shows the plot of the quantity $\mathcal{I}$ from Eq. (9) with respect to the post-selection angle $\theta$ of the linearly polarized post-selection state $|f\rangle=\cos \theta|H\rangle+\sin \theta|V\rangle$ with $|s\rangle=(|H\rangle-|V\rangle) / \sqrt{2}$. By choosing $\theta=0.18 \pi$ the value $\mathcal{I}^{(\exp )}=0.063(11)$, in agreement with quantum-mechanical prediction and at a distance of $5.7 \sigma$ from the noncontextual bound.

To demonstrate the validity of Eq. (7), the polarizer realizing $\mathcal{M}_{f}$ is removed, so that the probability $\xi_{\psi}(x)$ that a single photon prepared in any polarization state $|\psi\rangle$ is detected at the position $x$ after the interaction could be estimated. $\xi_{\psi}(x)$ is a faithful estimation of $P\left(x \mid \mathcal{P}, \mathcal{M}_{W}\right)$. This has been accomplished by by sending 


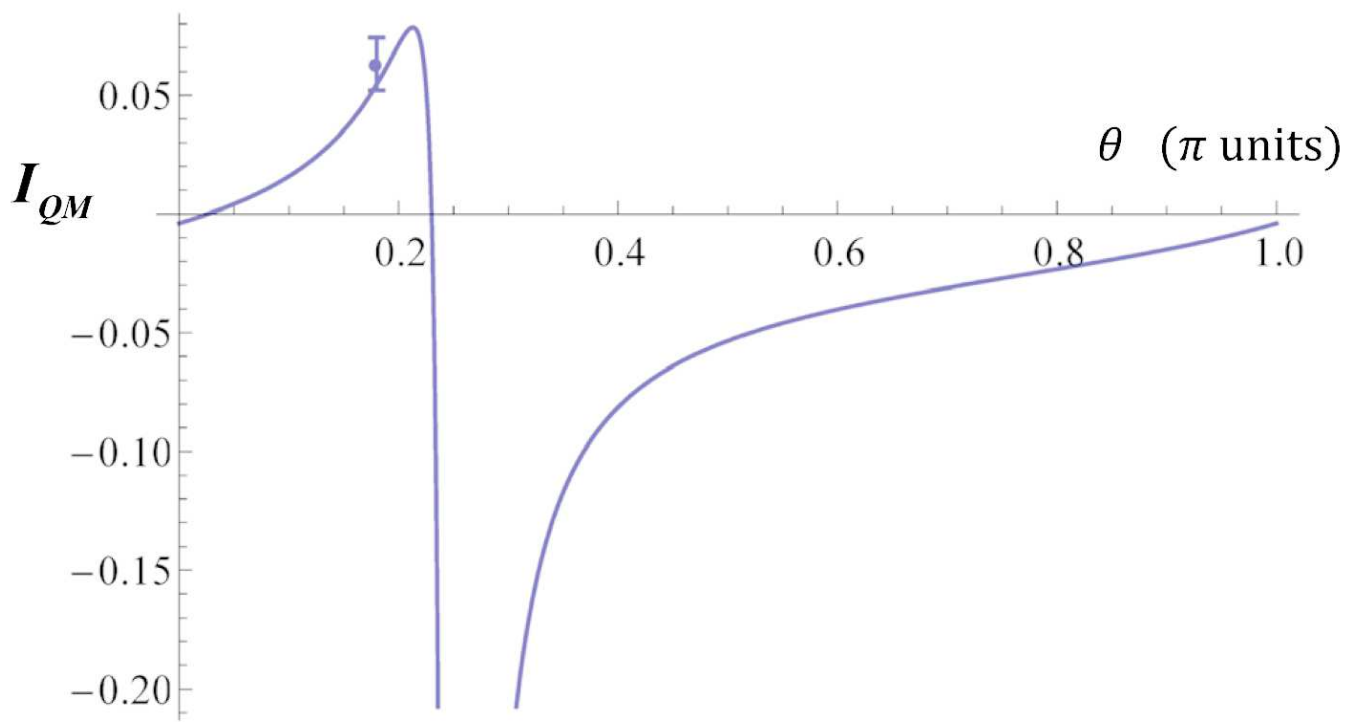

Figure 2: $\mathcal{I}$ with respect to the post-selection angle $\theta$. For $\theta=0.18 \pi$, a value $\mathcal{I}^{(\exp )}=0.063(11)$ has been observed, certifying a violation of the noncontextual bound of $5.7 \sigma$.

a tomographically complete set of four input states: $\{|H\rangle,|V\rangle,|+\rangle=(|H\rangle+|V\rangle) / \sqrt{2},|R\rangle=(|H\rangle-i|V\rangle) / \sqrt{2}\}$ and measuring $\xi_{\psi}(x)$ without the polarizer performing the post-selection. Then, $\xi_{\psi}(x)$ has been compared with the expected one obtained from the right side of Eq. (7), where the function $p_{n}(x)$ is reconstructed via a fit of the spatial profile in the absence of the weak interaction $\left(P\left(1 \mid \mathcal{P}, \mathcal{M}_{A}\right)=0\right)$ and the value of $g$ is estimated by maximizing the interaction $\left(P\left(0 \mid \mathcal{P}, \mathcal{M}_{A}\right)=0\right)$.

The fideliy between the measured $\xi_{\psi}(X)$ and the expected $\xi_{\psi}^{e}(x)$, evaluated by sampling more than 230 points in the region where $\xi_{\psi}(x)$ is significantly nonzero, for the four input states $|H\rangle,|V\rangle,|+\rangle$ and $|R\rangle$ is $0.997,0.991$, 0.994 and 0.996 , respectively. This shows the validity of this approach. The quality of the reconstruction is confirmed by a pixel-by-pixel proximity test of the two probability distributions for the pixels where the $\xi_{\psi}(x)$ is significantly nonzero. The proximity is defined as:

$$
\operatorname{Pr}_{\psi}(x)=\left[\frac{2 \xi_{\psi}(x) \xi_{\psi}^{e}(x)}{\left[\xi_{\psi}(x)\right]^{2}+\left[\xi_{\psi}^{e}(x)\right]^{2}}\right]^{\frac{1}{2}}
$$

Results are shown in Fig. 3.

Finally, it can be proved that the condition in Eq. (8) is fulfilled by using a method based on the comparison between experimental probabilities collected in different conditions, to get rid of any possible bias due to quantum-mechanical assumptions. At first, a tomographically complete set of states is prepared and the detection probabilities $P$ and $\bar{P}$, obtained with the Glan polarizer projecting the single photon states onto $|f\rangle$ and its orthogonal $|\bar{f}\rangle$, are registered. For each input state $|s\rangle$, these probabilities are given by $P=N^{f} / N_{T}$ and $\bar{P}=N^{\bar{f}} / N_{T}, N_{T}$ being the trigger counts and $N^{f(\bar{f})}$ the number of photon counts. Second, in order to nullify the weak interaction without altering the optical losses in the system, the position of the preparation stage and the birefringet crystals is switched, and then the same set of acquisition is performed. For each input state, these two acquisition correspond, respectively, to $P\left(O K \mid \mathcal{P}, \mathcal{M}_{W}, \mathcal{M}_{f}\right)$ and $P\left(O K \mid \mathcal{P}, \mathcal{M}_{f}\right)$ from Eq. (8). The last one is $P\left(O K \mid \mathcal{P}, \mathcal{M}_{d}\right)$, which is connected to the unknowk measurement procedure $\mathcal{M}_{d}$. It can be noticed that, by definition, $P\left(O K \mid \mathcal{P}, \mathcal{M}_{d}\right) \in[0,1]$, then:

$$
\left(1-p_{d}\right) P\left(O K \mid \mathcal{P}, \mathcal{M}_{f}\right) \leq P\left(O K \mid \mathcal{P}, \mathcal{M}_{W}, \mathcal{M}_{f}\right) \leq\left(1-p_{d}\right) P\left(O K \mid \mathcal{P}, \mathcal{M}_{f}\right)+p_{d}
$$



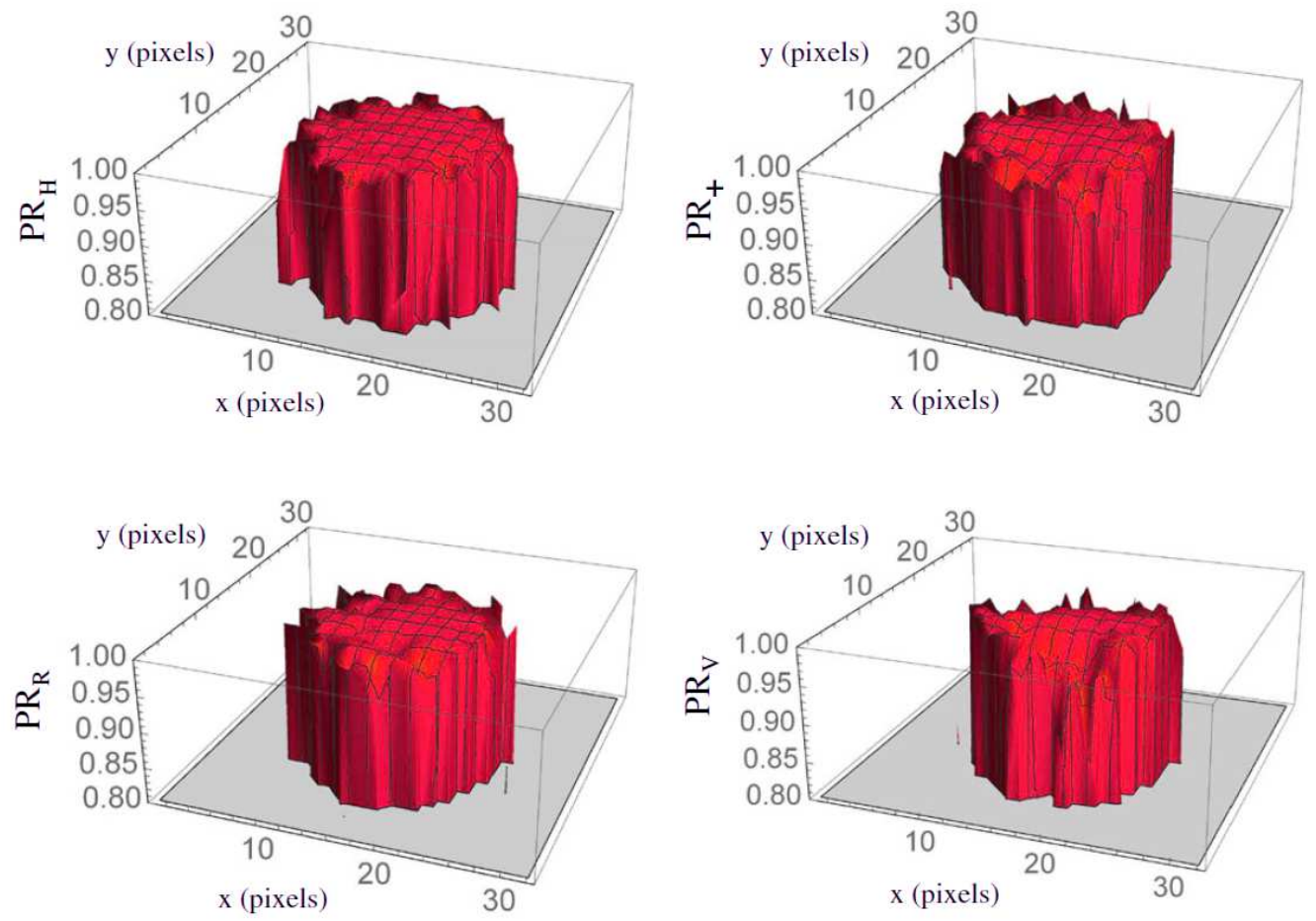

Figure 3: $\operatorname{Pr}_{\psi}(x)$ evaluated for the four input states $|H\rangle,|V\rangle,|+\rangle$ and $|R\rangle$. For all of them the proximity is above 0.99 , proving that the condition in Eq. (7) is satisfied.

which gives a lower and a upper bound to the parameter $p_{d}$ that are, for the collected data: $(0.000021(14)) \leq$ $p_{d} \leq(0.086(50))$, in agreement with the value derived by the system parameters (see Eq. $\left.(11)\right): p_{d}=0.0019(2)$.

Another consistency check can be performed by testing the output state after the sharp measurement $\mathcal{M}_{f}$, realized by the Glan polarizer, by inserting the tomographic apparatus in the setup, implicitly accepting some quantum mechanical assumptions. With this apparatus, two experiments can be performed.

In the first one, the apparatus is used to project the state after $\mathcal{M}_{f}$ onto $|f\rangle$ and $|\bar{f}\rangle$. A clear signal can be seen if the tomographic apparatus realizes the same projection as the Glan polarizer $(|f\rangle)$, but, when the tomographer projects onto $|\bar{f}\rangle$, the amount of signal registered is so small that it is completely indistinguishible from the detector noise, as expected from two subsequent projection onto orthogonal states. This confirms that the measurement process $\mathcal{M}_{f}$ is performing a projection onto the state $|f\rangle$.

In the second one, the state is tomographically reconstructed after the post-selection on $|f\rangle$. A tomographically complete set of input states $(|H\rangle,|+\rangle,|L\rangle=(|H\rangle+i|V\rangle) / \sqrt{2},|R\rangle)$ is produced and used to reconstruct via quantum tomography the state after the $\mathcal{M}_{f}$ measurement process. Fidelities of the tomograpic reconstruction with respect to the chosen $|f\rangle$ are: $\mathcal{F}_{H}=0.9995, \mathcal{F}_{+}=0.9999, \mathcal{F}_{L}=0.9991, \mathcal{F}_{R}=0.9811$. These values led to a estimate $p_{d}=0.0051(46)$, fitting the range obtained in Eq. (13) and in good agreement with the $p_{d}$ value derivated from the system experimental parameters $\left(p_{d}=0.0019(2)\right)$.

Since all the conditions of the theorem have been verified, it can be assessed that the results of this experiment clearly violate the noncontextual bound for the quantity $\mathcal{I}$ in Eq. (9), providing a demonstration of the connection between anomalous weak values and the contextual nature of quantum mechanics. 


\section{MEASURING INCOMPATIBLE OBSERVABLES BY EXPLOITING SEQUENTIAL WEAK VALUES}

Heisenberg uncertainty principle plays a fundamental role in quantum mechanics and quantum measurements. In particular, a crucial feature due to it is that measuring one observable completely erases the information on its conjugate one.

Within the weak measurement framework, the impossibility of measuring non-commuting observables can be partially relaxed.

In quantum metrology, weak values allows high-precision measurements (at least in presence of specific noises), as the tiny spin Hall effect or small beam detections and characterization of wavefunction. However, sequential weak values, which are more sensitive to the system's dynamics and whose time order is crucial, had not been performed before. One of the most interesting properties of sequential weak values is that they allow the simultaneous measurement of non-commuting observables on the same quantum state. Here we reach this result, measuring at the same time non-compatible polarizations by exploiting real single photons.

In our experiment, ${ }^{29}$ measurements of sequential weak values of two non-commutant observables $\hat{A}$ and $\hat{B}$ are obtained by means of two different couplings $\left(g_{x}\right.$ and $\left.g_{y}\right)$ to two distinct pointer observables (in our experiment the two transverse momenta $\hat{P}_{x}$ and $\hat{P}_{y}$ ) between the pre- and post-selection of the state. In particular, if we have a sequence of two weak interactions, e.g. the first interaction is described by the unitary transformation $U_{y}=\exp \left(-i g_{y} \hat{B} \otimes \hat{P}_{y}\right)$ and the second by $U_{x}=\exp \left(-i g_{x} \hat{A} \otimes \hat{P}_{x}\right)$, when measuring $\langle\hat{X} \hat{Y}\rangle$ we obtain:

$$
\langle\hat{X} \hat{Y}\rangle=\frac{1}{2} g_{x} g_{y} \operatorname{Re}\left[\langle\hat{A} \hat{B}\rangle_{w}+\langle\hat{A}\rangle_{w}^{*}\langle\hat{B}\rangle_{w}\right]
$$

Here, the result is proportional to the correlation between displacements inducted by meters $\hat{X}$ and $\hat{Y}$. I.e. it is related to the weak values of the operators $\hat{A}$ and $\hat{B}$, as well as the temporal correlation between them. Furthermore, when $\hat{A}$ and $\hat{B}$ are non-commuting, the product $\hat{A}$ and $\hat{B}$ is non-Hermitian, and time evolution will be, in the traditional quantum measurement framework, non-unitary. Instead, in our approach the two separate weak couplings induce unitary evolution in time. Thus, $\operatorname{Re}\left[\langle\hat{A} \hat{B}\rangle_{w}\right]$ can be extrated by measuring $\langle\hat{X} \hat{Y}\rangle$ and by evaluating each weak value independently, i.e. $\langle\hat{A}\rangle_{w}$ and $\langle\hat{B}\rangle_{w}$ (these can be obtained by measuring the mean values of the positions and momenta $\langle\hat{X}\rangle,\langle\hat{Y}\rangle,\left\langle\hat{P}_{x}\right\rangle$ and $\left.\left\langle\hat{P}_{y}\right\rangle\right)$.

In our sequential weak measurement implementation, the operators $\hat{A}$ and $\hat{B}$ are the polarization projectors $\Pi_{\psi}=|\psi\rangle\langle\psi|$ and $\Pi_{V}=|V\rangle\langle V|$, with $|\psi\rangle=\cos \theta|H\rangle+\sin \theta|V\rangle$. In our setup, a (heralded) single photon is preselected in the initial state $\left.\left|\phi_{i}\right\rangle\right\rangle=|s\rangle \otimes\left|r_{x}\right\rangle \otimes\left|r_{y}\right\rangle$, where $\left|s_{i}\right\rangle=\cos \theta_{i}|H\rangle+\sin \theta_{i}|V\rangle$ and $\left|r_{\xi}\right\rangle=\int \mathrm{d} \zeta \mathcal{F}_{\xi}(\zeta)|\zeta\rangle$, in which $\left|\mathcal{F}_{\xi}(\zeta)\right|^{2}$ is the probability density function of detecting the photon in the position $\xi=x, y$ of the transverse spatial plane. In our case, for the preselected single photons, the spatial distributions in both $x$ and $y$ axes are reasonably Gaussian and centered in zero with standard deviation $\sigma$.

When the two sequential weak interactions occur, single photons undergo spatial displacement both for $x$ and $y$ directions according to the unitary transformations $U_{y}=\exp \left(-i g_{y} \hat{\Pi}_{V} \otimes \hat{P}_{y}\right)$ and $U_{x}=\exp \left(-i g_{x} \hat{\Pi}_{\psi} \otimes \hat{P}_{x}\right)$. Afterwards, the single photons are post-selected in the linear polarization state $\left|\psi_{f}\right\rangle$ and detected by a spatialresolving detector. Therefore, the post-selected single-photon state is $\left.\left.\left|\phi_{f}\right\rangle\right\rangle=\left\langle\psi_{f}\left|\hat{U}_{x} \hat{U}_{y}\right| \phi_{i}\right\rangle\right\rangle$.

We are focusing on linear polarisations only, i.e. only real-valued weak values. Thus, we can evaluate the sequential weak value $\left\langle\hat{\Pi}_{\psi} \hat{\Pi}_{V}\right\rangle_{w}$, where in general the two projectors $\hat{\Pi}_{\psi}$ and $\hat{\Pi}_{V}$ are non-commuting operators. Indeed, from Eq.(14), we have $\langle\hat{X} \hat{Y}\rangle=\frac{1}{2} g_{x} g_{y}\left(\left\langle\hat{\Pi}_{\psi} \hat{\Pi}_{V}\right\rangle_{w}+\left\langle\hat{\Pi}_{\psi}\right\rangle_{w}\left\langle\hat{\Pi}_{V}\right\rangle_{w}\right),\langle\hat{X}\rangle=g_{x}\left\langle\hat{\Pi}_{\psi}\right\rangle_{w}$ and $\langle\hat{Y}\rangle=$ $g_{y}\left\langle\hat{\Pi}_{V}\right\rangle_{w}$. Note that $\langle\hat{X}\rangle$ and $\langle\hat{Y}\rangle$ are real because in our setup we use only linear polarizers. We stress that the relation between position mean values and polarisation weak values can exist only in a weak-interaction regime, i.e. only for $g / \sigma \ll 1$ : e.g. $g_{x} / \sigma \sim g_{y} / \sigma \sim 0.15$ in our case.

A scheme of the experimental setup in shown in Fig. 4.

The main results of this work are exposed in Fig. 5. We choose the following pre- and post-selected states: 


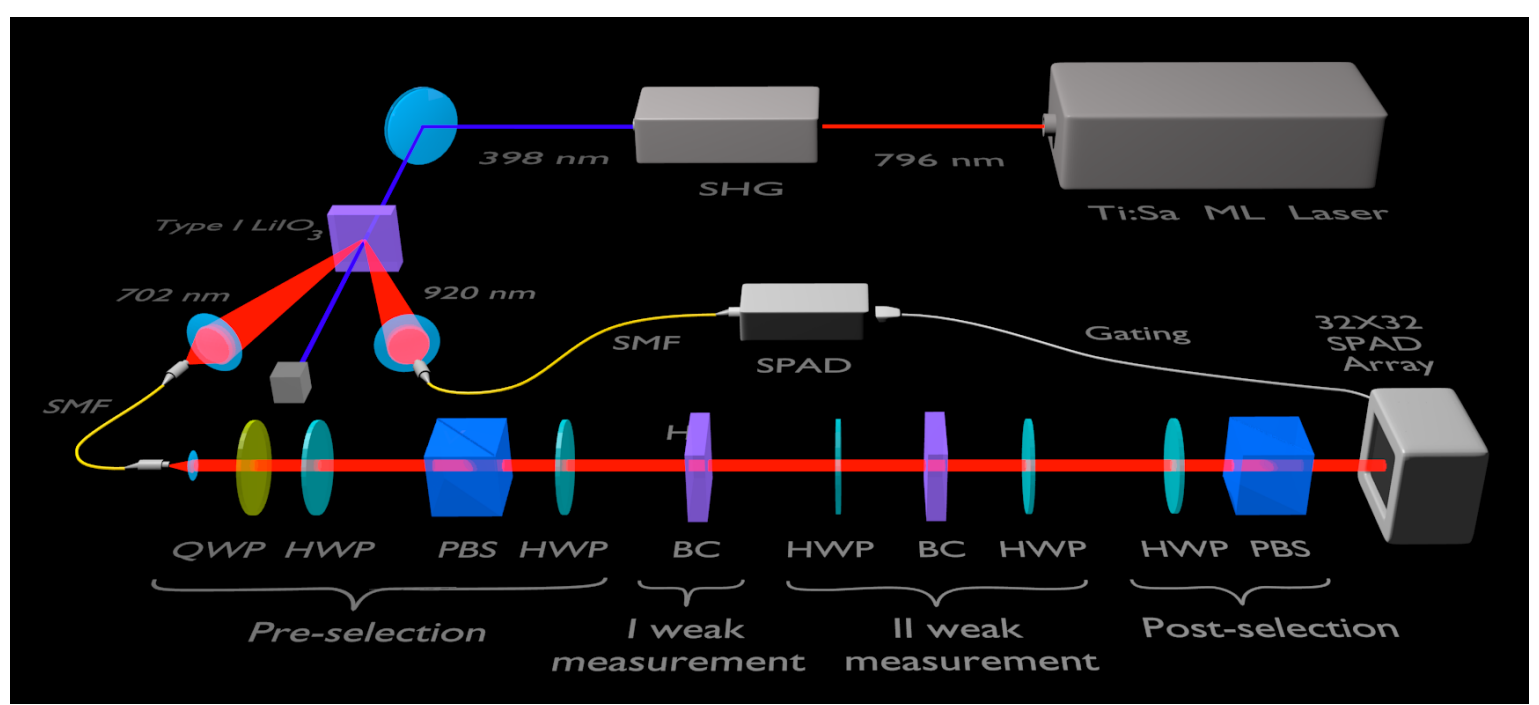

Figure 4: Experimental setup: a mode-locked laser with wavelenght $796 \mathrm{~nm}$ is frequency-doubled by means of second harmonic generation (SHG) and injected to a Lithium Iodate crystal $\left(\mathrm{LiIO}_{3}\right)$, where type-I parametric down-conversion (PDC) occurs. Idler photon $(920 \mathrm{~nm})$ is fiber-coupled and sent by a single-mode optical fiber (SMF) in a Silicon Single-Photon Avalanche Diode (Si-SPAD). Signal photon (702 nm) is SMF-coupled and then re-emitted in the open air path where the experiment takes place, with a Gaussian spatial distribution. There, it is pre-selected by a polarizing beam-splitter (PBS) followed by a half-wave plate (HWP). Each weak interaction is induced by a pair of birefringent crystals. In each pair, the first crystal is $2 \mathrm{~mm}$ thick and it has the optical axis with an angle of $45^{\circ}$ with respect to the propagation direction of the photons. The second one is $1.1 \mathrm{~mm}$ thick, with the optical axis orthogonal to the path of the photons. We need the second birefringent crystal in order to remove any relative phase and temporal walk-off induced by the first one. After the first weak measurement $\left(\mathrm{BC}_{V}\right)$, the polarization state of the signal photon is rotated by a $\mathrm{HWP}$, in order to perform the second weak measurement $\left(\mathrm{BC}_{H}\right)$ by means of a pair of birefringent crystals with optical axes orthogonal with respect to the corresponding crystals in the first weak measurement. Afterwards, the effect of the previous HWP is counterbalanced by another HWP, and finally a HWP and a PBS realize the post-selection on the polarization quantum state $|f\rangle$. At the end of the optical path, signal photon is detected by the same 32x32 SPAD array presented before (Fig. 1)

$|s\rangle=0.588|H\rangle+0.809|V\rangle$ and $\left|\psi_{f}\right\rangle=|H\rangle$ in Fig 5.a, and $|s\rangle=0.509|H\rangle+0.861|V\rangle$ and $\left|\psi_{f}\right\rangle=-0.397|H\rangle+$ $0.918|V\rangle$ in Fig 5.b. Here we plot the two weak values and the sequential one as a function of the angle $\theta$ of the polarization projector $\hat{\Pi}_{\psi}$ of the second weak interaction, all in good agreement with the theoretical predictions.

A paradoxical situation may be the case when one of the two single weak values is zero (within the uncertainty), while the sequential weak value of the two non-commuting observables is signicantly different from zero. E.g., in Fig. 5.a, with $\theta=0.2 \pi$, we obtain $\left\langle\hat{\Pi}_{V}\right\rangle_{w}=0.03 \pm 0.03,\left\langle\hat{\Pi}_{\psi}\right\rangle_{w}=1.44 \pm 0.04$, and $\left\langle\hat{\Pi}_{V} \hat{\Pi}_{\psi}\right\rangle_{w}=0.69 \pm 0.15$. For $\theta=0.9 \pi$, we obtain $\left\langle\hat{\Pi}_{V}\right\rangle_{w}=0.04 \pm 0.03,\left\langle\hat{\Pi}_{\psi}\right\rangle_{w}=0.35 \pm 0.04$, and $\left\langle\hat{\Pi}_{V} \hat{\Pi}_{\psi}\right\rangle_{w}=-0.46 \pm 0.10$, i.e. a single weak value positive, another one almost null positive and a negative joint weak value two order ov magnitude larger than the product between the two previous one. We also obtain, in Fig. 5.b at $\theta=0.9 \pi$, the situation in wich one of the single weak values and the sequential weak value are both positive, while the other single weak value is negative: $\left\langle\hat{\Pi}_{V}\right\rangle_{w}=1.40 \pm 0.04,\left\langle\hat{\Pi}_{\psi}\right\rangle_{w}=-0.24 \pm 0.03$, and $\left\langle\hat{\Pi}_{V} \hat{\Pi}_{\psi}\right\rangle_{w}=0.28 \pm 0.10$.

Our results are a good demonstration of the "product rule" breakdown when weak values are concerned. More generally, looking at Fig. 5.a we can observe that, despite the fact that $\left\langle\hat{\Pi}_{V}\right\rangle_{w}$ is almost zero everywhere, we have that both the single weak value of the other non-commuting observable and the sequential one are significantly non-zero. Furthermore, for both of them we have observed anomalous weak values. In Fig. 5.a we observe $\left\langle\hat{\Pi}_{\psi}\right\rangle_{w}>1$ and $\left\langle\hat{\Pi}_{\psi}\right\rangle_{w}<0$, as well as $\left\langle\hat{\Pi}_{V} \hat{\Pi}_{\psi}\right\rangle_{w}<0$.

The uncertainties related to the weak values shown in the plots of Fig. 5 are obtained with the uncertainty 


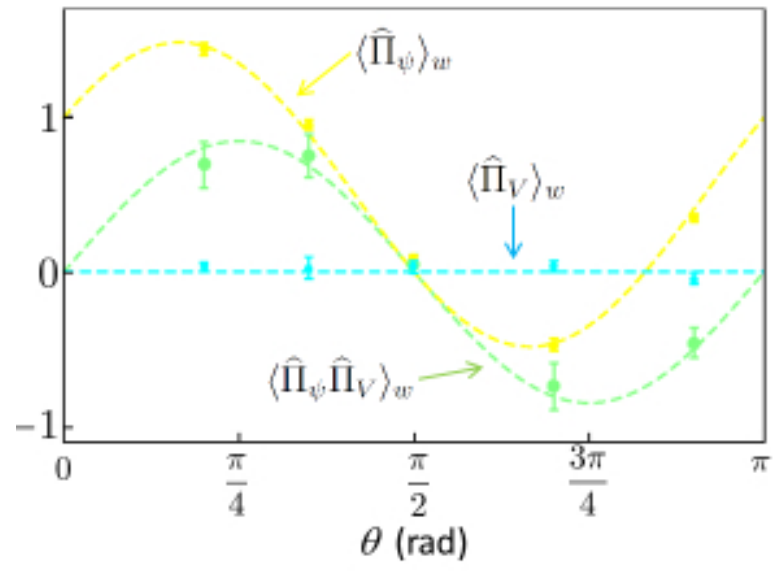

(a) $\theta=0.2 \pi$

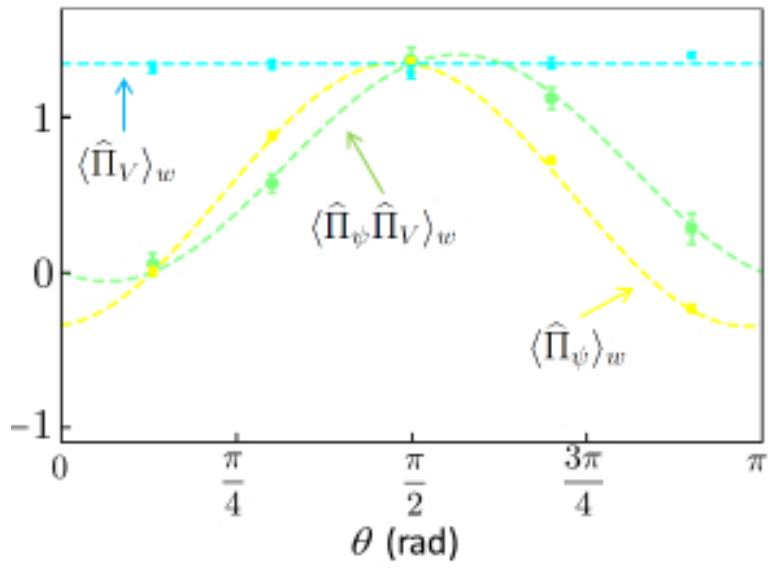

(b) $\theta=0.9 \pi$

Figure 5: Measured weak values (data points) compared with the theoretical predictions (dashed lines) for different $\widehat{\Pi}_{\psi}$ (i.e. for different values of $\theta$, since $|\psi\rangle=\cos \theta|H\rangle+\sin \theta|V\rangle$ ). Yellow and cyan points and lines correspond to the evaluations of the single-weak-value $\left\langle\widehat{\Pi}_{\psi}\right\rangle_{w}$ and $\left\langle\widehat{\Pi}_{V}\right\rangle_{w}$, respectively, while green points and line represent the evaluation of the sequential-weak-value $\left\langle\widehat{\Pi}_{\psi} \widehat{\Pi}_{V}\right\rangle_{w}$. Uncertainty bars are evaluated on the basis of sequences of repeated measurements. The uncertainty bars are naturally bigger in the case of the evaluation of sequential-weak-values with respect to the case single-weak-values, since in the former case the quantity measured is a covariance of positions, while in the latter cases they are position mean values. The pre-selected and post-selected states are respectively $\left|\psi_{i}\right\rangle=0.588|H\rangle+0.809|V\rangle$ and $\left|\psi_{f}\right\rangle=|H\rangle$ for plot (a), and $\left|\psi_{i}\right\rangle=0.509|H\rangle+0.861|V\rangle$ and $\left|\psi_{f}\right\rangle=-0.397|H\rangle+0.918|V\rangle$ for plot (b).

propagation standard rules (coverage factor $k=1$ ) starting from the images collected by our $32 \times 32$ SPAD array.

Here we demonstrate an unprecedented measurement capability, providing information on two non-commuting observables at the same time, as well as on the correlation between them, a feature forbidden in the conventional (i.e. POVM-based) measurement framework of quantum mechanics. In our sequential weak value experiment we exploit two weak couplings plus a "strong" post-selection measurement to obtain the simultaneous estimation of two single-operator weak values in connection with the same uncollapsed initial state, as well as the sequential weak value of two (in general non-commuting) observables. This is more significant than what can be obtained from a single weak interaction plus a strong post-selection measurement. Indeed, another weak value means more (non-counterfactual) information and interesting temporal correlations between non-commuting operators, including anomalous and paradoxical weak values. Sequential weak values can be used in direct measurement of the density matrix, ${ }^{30}$ and also in quantum process tomography, which makes use of this very technique of estimating an unknown dynamics without considerably changing it.

\section{ANOMALOUS WEAK VALUES AND THE VIOLATION OF A MULTIPLE-MEASUREMENT LEGGETT-GARG INEQUALITY}

The impossibility of interpreting the results of a measurement on quantum system in terms of pre-existing values is the topic of Bell's inequalities. ${ }^{31}$ This situation has also been studied by Leggett and Garg ${ }^{32}$ in the behaviour of macroscopic systems when one does subsequent measurements. For these objects, it is natural to assume that they will be found in a definite, realistic macro-state (macroscopic realism), and that a measurement, especially when it is achieved by a microscopic investigation, can not perturb such macro-state (non-invasive measurability). This consideration carried out a strong production of theoretical and experimental work about the inadequacy of such macro-realistic view; this has also inspired somehow the transposition of Bell's nonlocal argument to the time domain.

In its simplest form, Leggett and Garg's arrangement considers a macroscopic body undergoing three twooutcome measurements at different times, with the first being usually the preparation stage. The correlation 
among the outcomes can be shown not to be in accordance with macro-realistic prescriptions. The same scheme can also be employed for observing so-called post-selected values: the value of the second observable is considered only on events chosen according to the outcome of the last measurement. This measurement in the middle can also be driven in the weak regime: while shot by shot it delivers only partial information on the observable, it still provides the correct expectation value on average. While post-selection on a following measurement is mostly harmless in classical statistics, post-selection in the weak regime can lead to anomalous values. When one allows for such a weak measurement to be performed in a Leggett-Garg test, then a direct connection can be established between the violation of macro-realism and the emergence of anomalous post-selected values.

The simplest LGT one can design involves three measurements, ${ }^{33}$ which we label as $I_{A} ; I_{B}$, and $I_{C}$; these are two-outcome observables which can take either the value +1 or -1 . The inequality writes:

$$
-3 \leq \mathcal{B}_{3}=\left\langle I_{A} I_{B}\right\rangle+\left\langle I_{B} I_{C}\right\rangle-\left\langle I_{A} I_{C}\right\rangle \leq 1
$$

The measurement of $I_{A}$ can be taken to coincide with the initial preparation in the state $\left|\psi_{A}\right\rangle$, hence one can assign the fixed value +1 for $I_{A}$ :

$$
-3 \leq \mathcal{B}_{3}=\left\langle I_{B}\right\rangle+\left\langle I_{B} I_{C}\right\rangle-\left\langle I_{C}\right\rangle \leq 1
$$

The connection with anomalous post-selected values of $I_{B}$ is established by considering the two instances $I_{C}=1$ and $I_{C}=-1$ separately, each with the respective occurrence probabilities $p_{C}(1), p_{C}(-1)$ :

$$
\mathcal{B}_{3}=\left\langle I_{B}\right\rangle+\left[{ }_{1}\left\langle I_{B}\right\rangle-1\right] p_{C}(1)-\left[{ }_{-1}\left\langle I_{B}\right\rangle-1\right] p_{C}(-1)
$$

where ${ }_{a}\left\langle I_{B}\right\rangle$ is the post-selected value of $I_{B}$, conditioned on the outcome a for $I_{C}$. Exploiting the relation:

$$
\left\langle I_{B}\right\rangle={ }_{1}\left\langle I_{B}\right\rangle p_{C}(1)+_{-1}\left\langle I_{B}\right\rangle p_{C}(-1)
$$

it is possible manipulate Eq.(17) as

$$
\mathcal{B}_{3}=1+2 p_{C}(1)\left({ }_{1}\left\langle I_{B}\right\rangle-1\right)
$$

Inserting the condition for the standard values of $\left\langle I_{B}\right\rangle$, one recovers the limits of the Leggett-Garg inequality. This connection can be extended to the multiplemeasurement LGT that, in the simplest scenario, considers four measurements, including state preparation $I_{A}$ :

$$
\left|\mathcal{B}_{4}\right|=\left|\left\langle I_{B}\right\rangle+\left\langle I_{B} I_{C}\right\rangle++\left\langle I_{C} I_{D}\right\rangle-\left\langle I_{D}\right\rangle\right| \leq 2
$$

The structure of this inequality looks like the familiar Clauser-Horne-Shimony-Holt test for space-like separated systems; in that case, two partners alternate four distinct experimental arrangements, and verify whether the collected statistics is compatible with a local, realistic theory. Our situation can be viewed as a single system interrogated at four different times, including preparation. We can manipulate the four-measurement term $\mathcal{B}_{4}$ as we did for its three-measurement counterpart, by distinguishing the two instances for the last measurement $I_{D}$

$$
\left|\mathcal{B}_{4}\right|=\left|\left\langle I_{B}\right\rangle+\left\langle I_{B} I_{C}\right\rangle+p_{D}(1)\left[{ }_{1}\left\langle I_{C}\right\rangle-1\right]-p_{D}(-1)\left[-1\left\langle I_{C}\right\rangle-1\right]\right|
$$

We now assume that the post-selected values are bound to be found in the same ranges as the standard values: in this case, it is easy to verify that $\left|\mathcal{B}_{4}\right|$ is upper bounded by 2 . Differently from the three-measurement case, where any anomalous value would result in a violation, it can be shown that the inequality (21) demands a minimal value ${ }_{-1}\left\langle I_{C}\right\rangle \geq \frac{3-M}{2 p_{D}(-1)}$, where $M=\left\langle I_{B}\right\rangle+\left\langle I_{B} I_{C}\right\rangle+\left\langle I_{C}\right\rangle$, with a similar expression holding for ${ }_{1}\left\langle I_{C}\right\rangle$

In the last experiment, we exploit sequential weak value in order to implement a Leggett-Garg test (LGT). We obtained an explicit link between anomalous weak values of both individual observables and their correlation. Our experiment confirms the intimate connection between the observation of anomalies in the post-selected statistics of quantum measurement, and the failure of a macrorealistic view.

We perform a test of the inequality (21) by exploiting single photons undergoing sequential weak measurements of their polarization. Single photons are emitted by a downconversion source; at a heralding rate around 
$130 \mathrm{kHz}$, the quality of the emission is certified by a measured value of the antibunching parameter of $0.13 \pm 0.01$ without any background/dark-count subtraction. This implies that in our test we can genuinely associate the outcomes of the measurements to properties of single particles, avoiding classical wave-like analogies.

The experimental setup is the same shown in Fig. 4. The use of a single-mode fibre (SMF) allows to prepare the transverse profile $\mathcal{F}(x, y)$ in a Gaussian shape of width $\sigma$, which ensures that the two directions can be used as distinct pointers for the weak measurements. As shown in Fig. 4, the state of the photon is prepared (preselected) in the polarisation state $\left|\psi_{A}\right\rangle=\cos \alpha|H\rangle+\sin \alpha|V\rangle$ by means of a calcite polarising beam splitter (PBS) and a half-wave plate (HWP). These operations are implemented by coupling the polarisation to the transverse position by means of the unitary transformations $\hat{U}_{x}=\exp \left(-i g_{x} \hat{I}_{B} \otimes \hat{P}_{x}\right)$ and $\hat{U}_{y}=\exp \left(-i g_{y} \hat{I}_{C} \otimes \hat{P}_{y}\right)$, where $\hat{I}_{C}=|H\rangle\langle H|-| V\rangle\langle V|$ and $\hat{I}_{B}=\left|\psi_{\gamma}\right\rangle\left\langle\psi_{\gamma}|-| \psi_{\gamma}^{\perp}\right\rangle\left\langle\psi_{\gamma}^{\perp}\right|$ is associated to an arbitrary direction for the linear polarisation $\left|\psi_{\gamma}\right\rangle=\cos \gamma|H\rangle+\sin \gamma|V\rangle$ and $\left|\psi_{\gamma}^{\perp}\right\rangle=\sin \gamma|H\rangle-\cos \gamma|V\rangle$. The operators $\hat{P}_{x}$ and $\hat{P}_{y}$ are the momenta associated to the $x$ and $y$ directions, respectively.

After the second weak interaction, the photons arrive to a HWP that undoes the preceding rotation and, at the same time, determines the projection of the state onto one of the post-selected states $\left\langle\psi_{A}\right|,\left\langle\psi_{D}\right|=$ $\cos \delta\langle H|+\sin \delta\langle V|$ or $\left\langle\psi_{D}^{\perp}\right|=\sin \delta\langle H|-\cos \delta\langle V|$, by means of a PBS.

Since we are interested in the LGT as a tool for probing quantumness, we estimate each term in the inequality (21) separately in our setup. The chain of weak interactions and the space-resolved detector allow us to reconstruct the expectation values $\left\langle I_{B}\right\rangle$ and $\left\langle I_{C}\right\rangle$ by measuring the average $x$ and $y$ positions of the photons, respectively, when post-selecting on the input state $\left\langle\psi_{A}\right|:\langle\widehat{x}\rangle \simeq g_{x}\left\langle\widehat{I}_{B}\right\rangle$ and $\langle\widehat{y}\rangle \simeq g_{y}\left\langle\widehat{I}_{C}\right\rangle$. The covariance of the $x$ and $y$ positions gives $\langle\widehat{x} \widehat{y}\rangle \simeq \frac{g_{x} g_{y}}{2}\left(\left\langle\widehat{I}_{B} \widehat{I}_{C}\right\rangle+\left\langle\widehat{I}_{B}\right\rangle\left\langle\widehat{I}_{C}\right\rangle\right)$. By inverting these relations, it is possible to obtain the single and sequential values $\left\langle\widehat{I}_{C}\right\rangle,\left\langle\widehat{I}_{B}\right\rangle$ and $\left\langle\widehat{I}_{B} \widehat{I}_{C}\right\rangle$, estimated as weak averages. This resolves a major difficulty, in that by using standard "strong" measurements one would only have access to the symmetrized quantity $\frac{1}{2}\left\langle\psi_{A}\left|I_{B} I_{C}+I_{C} I_{B}\right| \psi_{A}\right\rangle .^{29}$ Post-selection on $\left\langle\psi_{D}\right|$ and $\left\langle\psi_{D}^{\perp}\right|$ occurrence delivers the probabilities $p_{D}(1)=\left|\left\langle\psi_{D} \mid \psi_{A}\right\rangle\right|^{2}$ and $p_{D}(-1)=\left|\left\langle\psi_{D}^{\perp} \mid \psi_{A}\right\rangle\right|^{2}$, as well as the weak values ${ }_{1}\left\langle I_{C}\right\rangle$ and ${ }_{-1}\left\langle I_{C}\right\rangle$.

Fig. 6 reports a theoretical simulation showing the shape of $\mathcal{B}_{4}$ for four different values of $\gamma$, plotted vs. the parameters $\alpha$ and $\delta$ determining the pre- and post-selection states. Aside of the yellow part of the surface, indicating where the classical bound holds, for each $\gamma$ value one observes orange and/or magenta areas, corresponding to the $\mathcal{B}_{4}>2$ and $\mathcal{B}_{4}<-2$ violations respectively.

We tested the inequality for different choices of the initial state $\alpha$, of the orientation $\gamma$ of weak measurement, and of the final post-selection $\delta$ : the four combinations have been identified to deliver a violation (indicated by the blue arrow in each plot reported in Fig. 6) close to the maximal value, whose results are illustrated in Table 1. For each of the four tests performed, the experimental values of $\mathcal{B}_{4}$ are in excellent agreement with the theoretical expectations within the statistical uncertainties, granting for both the positive and negative values a classical bound violation between 3.4 and 4.4 standard deviations. ${ }^{34}$ In the table, we also report the measured weak values showing how anomalies, i.e. values outside the standard range -1 to 1 , do flag the violation of the Leggett-Garg inequality: this corroborates the intimate connection between the emergence of anomalous values and the failure of a realistic description. 

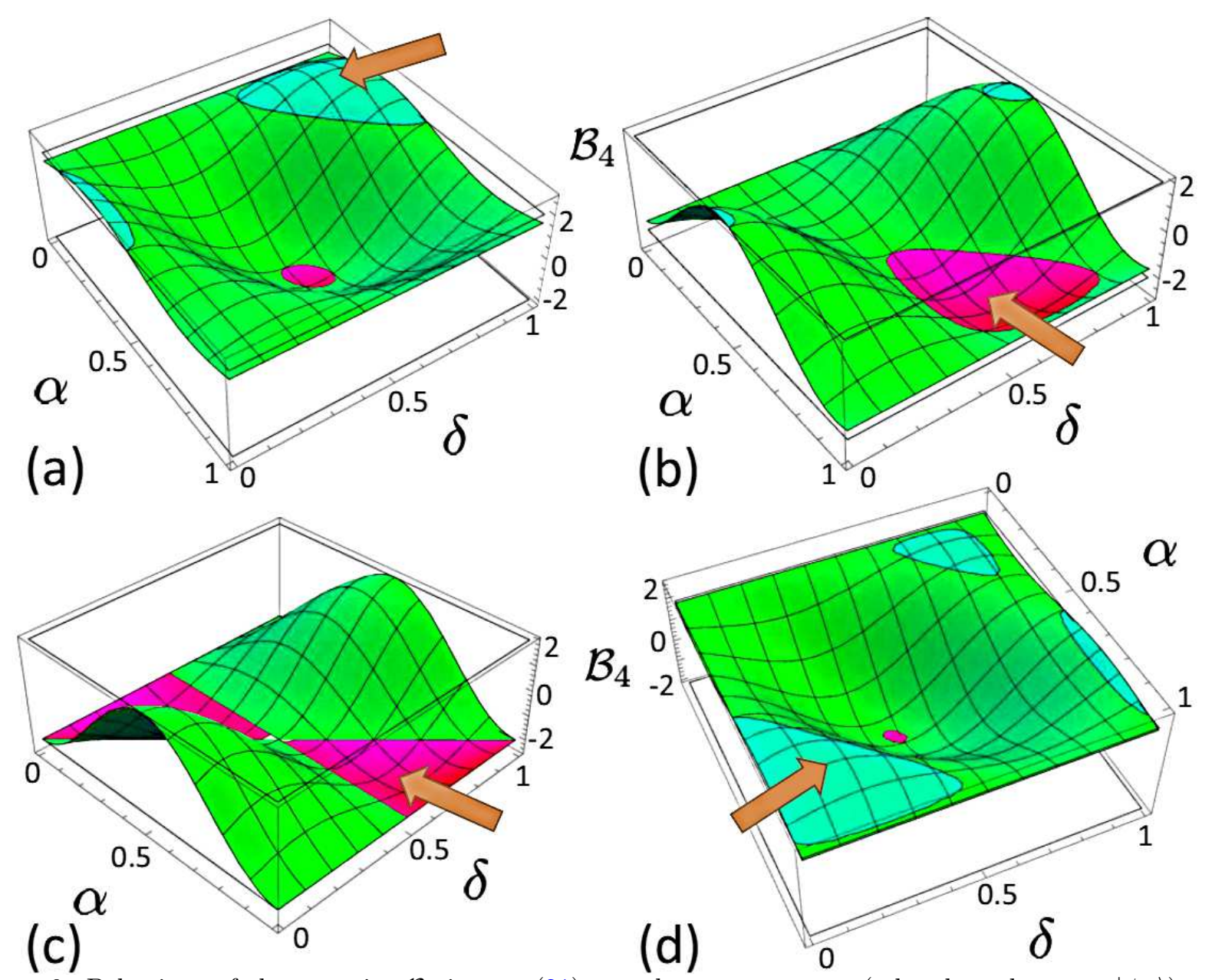

Figure 6: Behaviour of the quantity $\mathcal{B}_{4}$ in eq. (21) vs. the parameters $\alpha$ (related to the state $\left|\psi_{A}\right\rangle$ ) and $\delta$ (determining the states $\left|\psi_{D}\right\rangle$ and $\left|\psi_{D}^{\perp}\right\rangle$ ), both in $\pi$ units, for four different values of the parameter $\gamma$ defining the polarisation operator $I_{B}: \gamma=0.1 \pi$ for plot (a), $\gamma=0.4 \pi$ for plot (b), $\gamma=0.5 \pi$ for plot (c), $\gamma=0.95 \pi$ for plot (d). In each of these plots, the green part of the surface indicates the non-violation area $\left(-2 \leq \mathcal{B}_{4} \leq 2\right)$, while in cyan and magenta are highlighted respectively the positive $\left(\mathcal{B}_{4}>2\right)$ and negative $\left(\mathcal{B}_{4}<-2\right)$ violation areas. In each plot, the orange arrow indicates the point for which the violation was experimentally checked

\section{CONCLUSIONS}

In this paper we have shown three different experiment about quantum weak measurement. We demonstrated the crucial role of this measurement paradigm for onthological studying of non-contextuality of the wave function, showing that anomalous weak values violate the noncontextual bound, providing a demonstration of the connection between anomalous weak values and the contextual nature of quantum mechanics. Then, we demonstrated that weak measurements have the unprecedented measurement capability of providing information on two non-commuting observables at the same time, as well as on the correlation between them. Finally, we showed the importance of weak measurements for quantifying the quantumness of a state by showing that anomalous weak values flag the violation of the Leggett-Garg inequality.

Furthermore, weak measurements have paved the way to novel weak-interaction-based measurement paradigms. It is the case of Protective Measurements, originally proposed within the debate on the reality of the wave function, ${ }^{35}$ that combine weak measurements with some protection mechanism preserving the quantum state from decoherence. In their first experimental implementation, ${ }^{36}$ it has demonstrated how they can extract the expec- 


\begin{tabular}{|c|c|c|c|c|}
\hline Parameters & ${ }_{1}\left\langle I_{c}\right\rangle$ & $-1\left\langle I_{c}\right\rangle$ & $\mathcal{B}_{4}^{(t h)}$ & $\mathcal{B}_{4}^{(\text {exp })}$ \\
\hline $\begin{array}{c}\gamma=0.1 \pi \\
\alpha=0.233 \pi \\
\delta=0.867 \pi\end{array}$ & $2.34 \pm 0.04$ & $-0.34 \pm 0.04$ & 2.82 & $2.76 \pm 0.17$ \\
\hline $\begin{array}{c}\gamma=0.4 \pi \\
\alpha=0.767 \pi \\
\delta=0.633 \pi\end{array}$ & $-0.30 \pm 0.04$ & $2.20 \pm 0.04$ & -2.82 & $-2.74 \pm 0.18$ \\
\hline $\begin{array}{c}\gamma=0.5 \pi \\
\alpha=0.833 \pi \\
\delta=0.667 \pi\end{array}$ & $0.01 \pm 0.06$ & $1.86 \pm 0.06$ & -2.50 & $-2.56 \pm 0.16$ \\
\hline $\begin{array}{c}\gamma=0.95 \pi \\
\alpha=0.8 \pi \\
\delta=0.15 \pi\end{array}$ & $1.86 \pm 0.04$ & $-0.12 \pm 0.06$ & 2.71 & $2.86 \pm 0.19$ \\
\hline
\end{tabular}

Table 1: Leggett-Garg inequality violation results obtained in our four experimental scenarios. The first column reports the $\gamma, \alpha$ and $\delta$ values exploited in each experiment, the second and third columns host respectively the theoretical $\left(\mathcal{B}_{4}^{(t h)}\right)$ and experimentally-obtained $\left(\mathcal{B}_{4}^{(\exp )}\right)$ values of the quantity $\mathcal{B}_{4}$, while the fourth and fifth columns show the anomalous weak values obtained for $I_{C}$ in each experiment.

tation value of a quantum observable by measuring a single (protected) particle in a single experiment, something in sharp contrast with usual quantum (and even classical) measurement frameworks. In fact, we know that one description provides only probabilities for obtaining various eigenvalues of a quantum variable. The eigenvalues and the corresponding probabilities specify the expectation value of a physical observable, which is known to be a statistical property of an ensemble of quantum systems. In contrast to this paradigm, it has been demonstrated a method for measuring the expectation value of a physical variable on a single particle.

We believe that theese experiments will prove themselves useful both for studies of foundations of quantum mechanics and for the quantum technologies framework.

\section{REFERENCES}

[1] Aharonov, Y., Albert, D. Z., and Vaidman, L., "How the result of a measurement of a component of the spin of a spin-1/2 particle can turn out to be 100," Phys. Rev. Lett. 60, 1351-1354 (Apr 1988).

[2] Aharonov, Y., Cohen, E., and Elitzur, A. C., "Foundations and applications of weak quantum measurements," Phys. Rev. A 89, 052105 (May 2014).

[3] Aharonov, Y., Botero, A., Popescu, S., Reznik, B., and Tollaksen, J., "Revisiting hardy's paradox: counterfactual statements, real measurements, entanglement and weak values," Physics Letters A 301(3), 130 138 (2002).

[4] Aharonov, Y., Cohen, E., and Elitzur, A. C., "Can a future choice affect a past measurement's outcome?," Annals of Physics 355, 258 - 268 (2015).

[5] F. Piacentini, e. a., "Determining the quantum expectation value by measuring a single photon," Nature Physics 13, 1191-1194 (2017).

[6] G. Brida, M. G. and Piacentini, F., "Experimental local realism tests without fair sampling assumption," F. Eur. Phys. J. D 44, 577 (2007).

[7] G Brida, M Genovese, F. P., "Experimental local realism tests without fair sampling assumption," The European Physical Journal D 44, 577-580 (2007).

[8] Lev Vaidman, Alon Ben-Israel, J. D. L. K. M. W. J. M. C. S. R. B. H. W., "Weak value beyond conditional expectation value of the pointer readings," arXiv preprint: arXiv:1611.03989 (2016).

[9] Ritchie, N. W. M., Story, J. G., and Hulet, R. G., "Realization of a measurement of a "weak value"," Phys. Rev. Lett. 66, 1107-1110 (Mar 1991).

[10] Resch, K. J., "Amplifying a tiny optical effect," Science 319(5864), 733-734 (2008). 
[11] Schlosshauer, M., "Measuring the quantum state of a single system with minimum state disturbance," Phys. Rev. A 93, 012115 (Jan 2016).

[12] Y. Aharonov, J. Anandan, L. V., "Protective measurements," Found Phys 26(117) (1996).

[13] Fabrizio Piacentini, Alessio Avella, E. R. S. V. R. L. F. V. A. T. M. G. G. B. E. C. L. V. I. P. D. M. G., "Protective measurements: extracting the expectation value by measuring a single particle," (2018).

[14] Hosten, O. and Kwiat, P., "Observation of the spin hall effect of light via weak measurements," Science 319(5864), 787-790 (2008).

[15] Tamir, B. and Cohen, E., "Introduction to weak measurements and weak values," Quanta 2(1) (2013).

[16] Genovese, M., "Interpretations of quantum mechanics and the measurement problem," Adv. Sci. Lett 3, $249-258$.

[17] Fabrizio Piacentini, Alessio Avella, M. G. R. L. F. V. A. T. G. B. I. P. D. M. G., "Investigating the effects of the interaction intensity in a weak measurement," arXiv preprint arXiv:1709.04869 (2017).

[18] Dressel, J., Malik, M., Miatto, F. M., Jordan, A. N., and Boyd, R. W., "Colloquium: Understanding quantum weak values: Basics and applications," Rev. Mod. Phys. 86, 307-316 (Mar 2014).

[19] Genovese, M., "Research on hidden variable theories: A review of recent progresses," Physics Reports 413, 319-396 (2005).

[20] Pusey, M. F., "Anomalous weak values are proofs of contextuality," Phys. Rev. Lett. 113, 200401 (Nov 2014).

[21] G. Brida, I. P. Degiovanni, M. G. F. P. P. T. A. D. F. A. T. A. B. S. C. S. A. G. M. G. S. V. P. A. M. and Giudice, A., "An extremely low-noise heralded single-photon source: A breakthrough for quantum technologies," Appl. Phys. Lett. 101 (2012).

[22] Piacentini, F., Avella, A., Levi, M. P., Lussana, R., Villa, F., Tosi, A., Zappa, F., Gramegna, M., Brida, G., Degiovanni, I. P., and Genovese, M., "Experiment investigating the connection between weak values and contextuality," Phys. Rev. Lett. 116, 180401 (May 2016).

[23] Avella, A., "Theory of parametric down conversion in bulk non-linear crystals: An introduction," Journal of Advanced Physics 4(3), 252-262 (2015).

[24] Castelletto, S., Degiovanni, I. P., Schettini, V., and Migdall, A., "Spatial and spectral mode selection of heralded single photons from pulsed parametric down-conversion," Opt. Express 13, 6709-6722 (Sep 2005 ).

[25] Grangier, P., Roger, G., and Aspect, A., "Experimental evidence for a photon anticorrelation effect on a beam splitter: A new light on single-photon interferences," EPL (Europhysics Letters) 1(4), 173 (1986).

[26] F. Villa, R. Lussana, D. B. S. T. A. T. F. Z. A. D. M. D. C. D. D. S. W. W. B., "Cmos imager with 1024 spads and tdes for single-photon timing and 3-d time-of-flight," IEEE Journal of Selected Topics in Quantum Electronics 20, 364-373 (Nov 2014).

[27] Jeff Z. Salvail, Megan Agnew, A. S. J. E. B. J. L. . R. W. B., "Full characterization of polarization states of light via direct measurement," Nature Photonics 7, 316-321 (2013).

[28] Bogdanov, Y. I., Brida, G., Genovese, M., Kulik, S. P., Moreva, E. V., and Shurupov, A. P., "Statistical estimation of the efficiency of quantum state tomography protocols," Phys. Rev. Lett. 105, 010404 (Jul 2010).

[29] Piacentini, F., Avella, A., Levi, M. P., Gramegna, M., Brida, G., Degiovanni, I. P., Cohen, E., Lussana, R., Villa, F., Tosi, A., Zappa, F., and Genovese, M., "Measuring incompatible observables by exploiting sequential weak values," Phys. Rev. Lett. 117, 170402 (Oct 2016).

[30] Lundeen, J. S. and Bamber, C., "Procedure for direct measurement of general quantum states using weak measurement," Phys. Rev. Lett. 108, 070402 (Feb 2012).

[31] A Avella, M. G., "50 years of experimental bell inequalities: A short introduction," Journal of Advanced Physics 4, 233-235 (2015).

[32] Leggett, A. J. and Garg, A., "Quantum mechanics versus macroscopic realism: Is the flux there when nobody looks?," Phys. Rev. Lett. 54, 857-860 (Mar 1985).

[33] Barbieri, M., "Multiple-measurement leggett-garg inequalities," Phys. Rev. A 80, 034102 (Sep 2009 ).

[34] Avella, A., Piacentini, F., Borsarelli, M., Barbieri, M., Gramegna, M., Lussana, R., Villa, F., Tosi, A., Degiovanni, I. P., and Genovese, M., "Anomalous weak values and the violation of a multiple-measurement leggett-garg inequality," Phys. Rev. A 96, 052123 (Nov 2017). 
[35] Y. Aharonov, J. Anandan, L. V., "Meaning of the wave function," Phys. Rev. A 47, 4616 (1993).

[36] Fabrizio Piacentini, Alessio Avella, E. R. R. L. F. V. A. T. M. G. G. B. E. C. L. V. I. P. D. M. G., "Determining the quantum expectation value by measuring a single photon," Nature Physics 13, 1191?1194 (2017). 